\title{
GAS AND DUST IN INTERSTELLAR CLOUDS
}

\author{
R. D. DAVIES
}

University of Manchester, Nuffield Radio Astronomy Laboratories, Jodrell Bank, U.K.

\begin{abstract}
Detailed measurements have been made of the $\mathrm{H}, \mathrm{OH}$ and $\mathrm{H}_{2} \mathrm{CO}$ lines formed in the $\mathrm{L} 1534$ dense dust cloud; some $\mathrm{H}$ and $\mathrm{H}_{2} \mathrm{CO}$ observations have been made of the dust cloud lying behind the Orion cluster. These indicate a similar distribution of the 3 species throughout the dust clouds. Studies of $\mathrm{H}, \mathrm{OH}$ and $\mathrm{H}_{2} \mathrm{CO}$ absorption features in clouds lying in front of bright radio sources provide estimates of the physical conditions in regions of molecule formation.
\end{abstract}

\section{Introduction}

There are a number of situations in the interstellar medium where an association between gas and dust can be observed or directly inferred. One such situation is found in the dense dust clouds as listed for example in the Lynds (1962) catalogue. Here the molecule $\mathrm{OH}$ is seen in emission and the formaldehyde $\left(\mathrm{H}_{2} \mathrm{CO}\right)$ is an absorption against the $2.7 \mathrm{~K}$ background. Also the neutral hydrogen in many of these dust clouds is cool and can be observed in absorption against the galactic background of neutral hydrogen which is at a higher temperature. The other situation where gas and dust may be studied together is in the dense interstellar neutral hydrogen clouds which produce absorption when seen against strong continuum radio sources. These clouds also contain $\mathrm{OH}$ and $\mathrm{H}_{2} \mathrm{CO}$; their dust content can be inferred from the total optical obscuration in the line of sight to the optical counterpart of the radio source.

\section{Dense Dust Clouds}

Since the discovery of $\mathrm{OH}$ emission (Heiles, 1968) and $\mathrm{H}_{2} \mathrm{CO}$ absorption (Palmer et al., 1969 ) in dense dust clouds a number of investigations have been made of these objects. The present observations (Cohen et al., 1972) were aimed at obtaining detailed information about conditions of the neutral hydrogen in two such dust clouds.

One cloud lies in the Orion nebula region. It is the object in which Kutner and Thaddeus (1971) found $\mathrm{H}_{2} \mathrm{CO}$ in absoprtion in a region $\sim 0.5^{\circ}$ in extent. Our more extensive observations show $\mathrm{H}_{2} \mathrm{CO}$ absorption in an even larger area surrounding the Orion nebula. Examination of the Palomar Sky Survey prints shows a large obscuring cloud crossing the Orion nebula region. A preliminary study indicates 3-4 mag. of visual absorption in the cloud. When this is compared with the interstellar absorption in front of the Orion cluster which amounts to $\sim 0.5 \mathrm{mag}$. it is clear that this dense cloud lies behind the Orion cluster and nebula. This explains the absence of $\mathrm{H}_{2} \mathrm{CO}$ absorption in front of the Orion A continuum radio source. Neutral hydrogen measurements with the Mark IA radio telescope (beamwidth 11') show much fine structure 
in this area, some of which may be attributable to cool absorbing hydrogen in this dust cloud.

The dense dust cloud numbered 1534 in Lynd's catalogue (Cloud 2 in Heiles' nomenclature) has been the subject of much study in the radio lines of $\mathrm{H}, \mathrm{OH}$ and $\mathrm{H}_{2} \mathrm{CO}$ At Jodrell Bank an attempt is being made to compare the line profile in each of these species with similar beam widths. Hydrogen profiles have been taken with the Mark IA radio telescope (beamwidth $11^{\prime}$ ), $\mathrm{H}_{2} \mathrm{CO}$ profiles have been taken with the Mark II radio telescope (beamwidth $9^{\prime}$ ) and soon $\mathrm{OH}$ profiles will be obtained with the Mark IA $\left(9^{\prime}\right)$. The present comparison of the $\mathrm{H}$ and $\mathrm{H}_{2} \mathrm{CO}$ is made with $\mathrm{OH}$ spectra

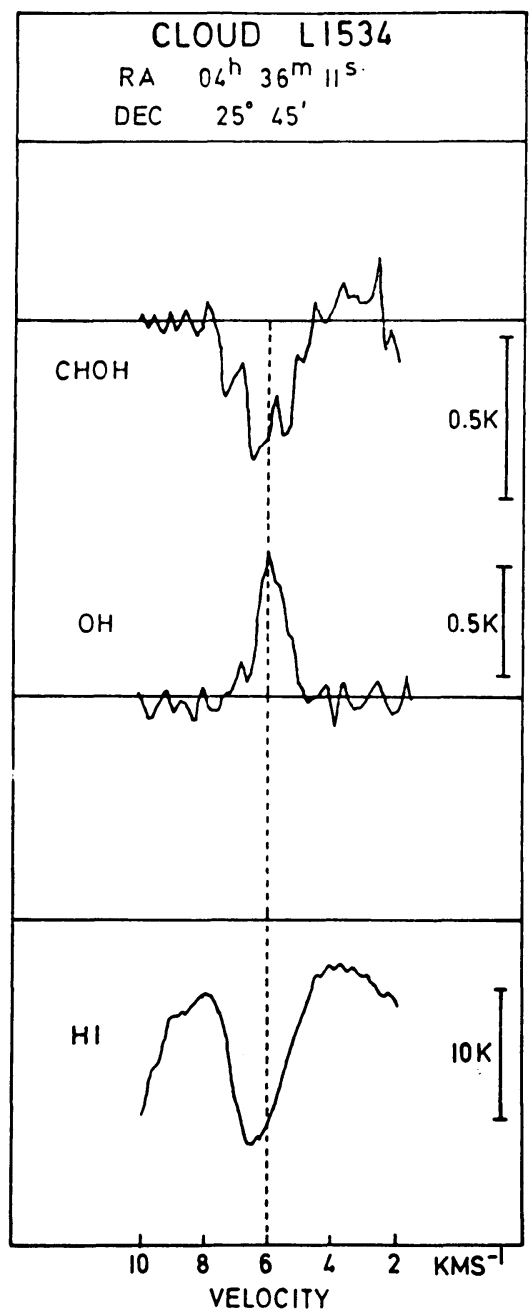

Fig. 1. $\mathrm{H}, \mathrm{OH}$ and $\mathrm{H}_{2} \mathrm{CO}$ spectra at $\mathrm{RA}=04^{\mathrm{h}} 36^{\mathrm{m}} 11^{\mathrm{s}}$, Dec $=25^{\circ} 45^{\prime}$ in cloud 1534 of Lynds' (1962) catalogue. The intensities are given as antenna temperatures. The structure in the $\mathrm{H}_{2} \mathrm{CO}$ spectrum is believed to be due to noise fluctuations only. Observing beamwidths for the $\mathrm{H}_{2} \mathrm{CO}, \mathrm{OH}$ and $\mathrm{H}$ lines are $9^{\prime}, 18^{\prime}$ and $11^{\prime}$ respectively. 
taken by Heiles (1970) with the NRAO $140 \mathrm{ft}$ telescope (beamwidth $18^{\prime}$ ). Spectra in the 3 lines are shown for the point RA $=04^{\mathrm{h}} 36^{\mathrm{m}} 11^{\mathrm{s}}$, Dec $=24^{\circ} 45^{\prime}$ in Figure 1 . The $\mathrm{H}_{2} \mathrm{CO}$ absorption is produced against the $2.7 \mathrm{~K}$ Universal blackbody background radiation. The $\mathrm{OH}$ emission is the 'normal' $1667 \mathrm{MHz}$ emission line. The neutral hydrogen profile shows narrowband absorption in a broader background emission profile; this absorption occurs only at the position of the dust cloud and is absent in the unobscured regions in the vicinity.

The central velocity of the features in each of the lines is closely similar which suggests that they arise in the same parts of the dust cloud. The width of the $\mathrm{OH}$ emission feature is less than that of the $\mathrm{H}$ and $\mathrm{H}_{2} \mathrm{CO}$ absorption feature. This difference may arise because the $\mathrm{OH}$ beam area is about a factor of 3 greater and may well be sampling a different velocity. This is likely because our $\mathrm{H}$ observations show significant velocity changes over distances of $10^{\prime}$ or so.

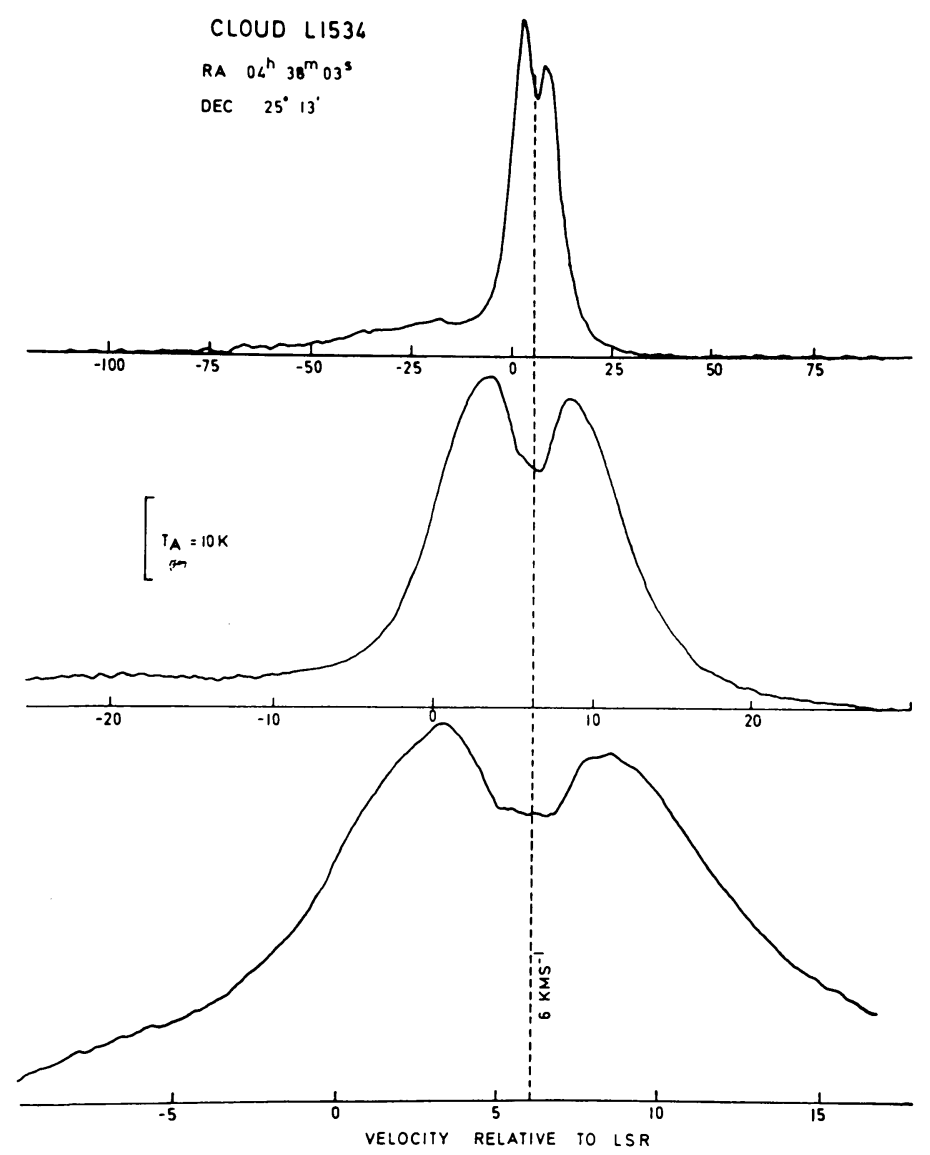

Fig. 2. Neutral hydrogen spectra at $R A=04^{\mathrm{h}} 38^{\mathrm{m}} 03^{\mathrm{s}}, \mathrm{Dec}=25^{\circ} 13^{\prime}$ in cloud 1534 of Lynd's (1962) catalogue. The velocity resolutions are (from top to bottom) $1.86,0.46$ and $0.23 \mathrm{~km} \mathrm{~s}^{-1}$. The integration times were increased in the narrower bandwidth observations to give similar rms noise on each spectrum. 
An estimate can be made of the kinetic temperature of the neutral hydrogen in L1534 by examining the thermal broadening in the absorption features. Many of the profiles exhibit structure which indicates the presence of more than one velocity feature. The H-line profile in Figure 1 is asymmetrical with its steepest edge on the high velocity side. Another example of structure in the $\mathrm{H}$-line absorption is shown in Figure 2 which illustrates the spectrum at $\mathrm{RA}=04^{\mathrm{h}} 38^{\mathrm{m}} 03^{\mathrm{s}}$, Dec $=25^{\circ} 13^{\prime}$ taken with different overall bandwidths and resolutions. At least two absorption components are present in this spectrum. An upper limit for the kinetic temperature derived from the broadening of such profiles is $20 \mathrm{~K}$, assuming the broadening is entirely thermal. Clearly macroscopic motions exist as revealed by the $\mathrm{OH}$ and $\mathrm{H}_{2} \mathrm{CO}$ spectra. Actual kinetic temperatures as low as $10 \mathrm{~K}$ would be compatible with the observations. Heiles estimated an excitation temperature of $5.5 \mathrm{~K}$ for the $\mathrm{OH}$ by a comparison of the 1665 and $1667 \mathrm{MHz}$ transitions. Theoretically this temperature is expected to lie between the kinetic temperature and the $2.7 \mathrm{~K}$ Universal background; its actual value depends upon the gas density. This $\mathrm{OH}$ excitation temperature is consistent with a kinetic temperature in the range $10-20 \mathrm{~K}$ inferred from the neutral hydrogen data.

The dense dust clouds are clearly regions of rich molecule production. The high dust content protects the molecules from dissociation or ionization by the interstellar ultraviolet flux; the high gas density ensures high reaction rates for molecule production (Hollenbach et al., 1971).

\section{Normal Absorbing Clouds}

A more common type of cloud in interstellar space wich contains molecules and dust is the cloud seen in absorption at neutral hydrogen wavelengths against continuum

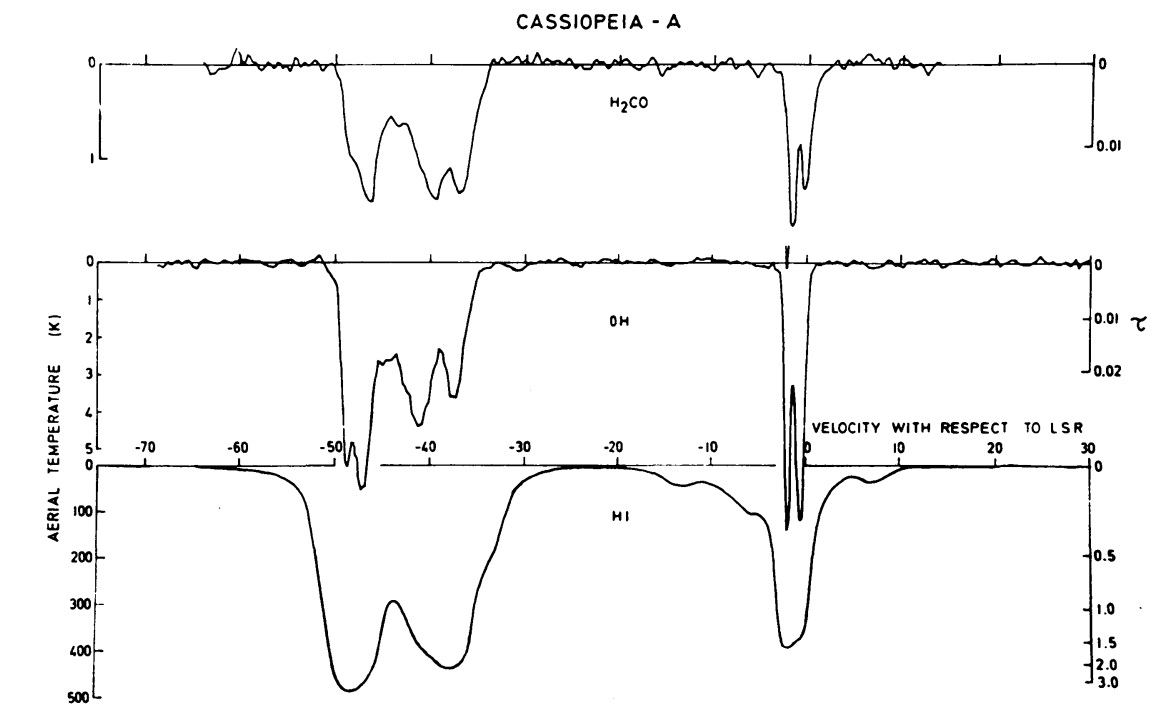

Fig. 3. The absorption spectra of Cassiopeia $\mathrm{A}$ in the lines of $\mathrm{H}, \mathrm{OH}$ and $\mathrm{H}_{2} \mathrm{CO}$ taken with the Mark II radio telescope. 
radio sources. An example is the group of clouds seen in absorption against Cassiopeia A shown in Figure 3. The $\mathrm{H}, \mathrm{OH}$ and $\mathrm{H}_{2} \mathrm{CO}$ spectra were all taken with the Mark II radio telescope. Although thermal broadening in the $\mathrm{H}$-line spectrum makes the identification of some components less certain, it is possible to see the stronger $\mathrm{H}$-line components in both the $\mathrm{OH}$ and $\mathrm{H}_{2} \mathrm{CO}$ spectra. The same situation is found in the absorption spectra of Cyg A and Tau A (Davies and Matthews, 1972).

An important parameter of these clouds, as regards the processes of molecule formation, is the amount of obscuring matter in each cloud. This determines the destruction rate of molecules by UV radiation. The optical absorption in front of the optical remnants of the supernovae Cas A and Tau, and of the extragalactic Cyg A optical object are given in the literature. The total obscuration through each of the clouds, is 0.4 to $0.8^{\mathrm{m}}$ assuming it is divided evenly amongst all the $\mathrm{H}$-line components. The mean parameters of the molecule bearing absorption clouds are given in Table I.

TABLE I

Parameters of molecule bearing absorption clouds

\begin{tabular}{ll}
$\int n(\mathrm{H}) \mathrm{d} l=N(\mathrm{H})$ & $1.1 \times 10^{21} \mathrm{~cm}^{-2}$ \\
Temperature $(\mathrm{H})$ & $50-100 \mathrm{~K}$ \\
$N(\mathrm{OH}) / N(\mathrm{H})$ & $1.1 \times 10^{-7}$ \\
$N\left(\mathrm{H}_{2} \mathrm{CO}\right) / N(\mathrm{H})$ & $2 \times 10^{-9}$ \\
Velocity dispersion & $0.74 \mathrm{~km} \mathrm{~s}^{-1}$ \\
Cloud diameter & $10 \mathrm{pc}$ \\
Mean neutral hydrogen density & $36 \mathrm{~cm}^{-3}$ \\
Mean cloud mass & $640 M_{\bigcirc}$ \\
Visual absorption at cloud centre & $0.2^{m}-0.4^{m}$ \\
Magnetic field & $2-20 \mu \mathrm{G}$ \\
\hline
\end{tabular}

The low obscuration in these clouds means that the lifetimes against photoinduced destructive reactions are $\sim 1000$ years for $\mathrm{OH}$ and $100 \mathrm{yr}$ for $\mathrm{H}_{2} \mathrm{CO}$. The lifetime of an interstellar cloud on the other hand is $\sim 10^{7} \mathrm{yr}$, the time between cloud collisions. Thus the molecular destruction time is $10^{-5}$ to $10^{-4}$ of the lifetime of the interstellar clouds. This argues strongly that the molecules should be continually formed. In fact a gas atom such as oxygen will collide with a dust grain on timescale similar to the destruction timescales mentioned above. However no satisfactory process for molecule formation, during the atom-grain encounter has yet been worked out.

\section{References}

Cohen, R. J., Davies, R. D., and Wilson, A. J.: 1972, in preparation.

Davies, R. D. and Matthews, H. E.: 1972, Monthly Notices Roy. Astron. Soc. 156, 253.

Heiles, C.: 1968, Astrophys. J. 151, 919.

Heiles, C.: 1970, Astrophys. J. 160, 51.

Hollenbach, D. J., Werner, M. W., and Salpeter, E. E.: 1971, Astrophys. J. 163, 165.

Kutner, M. and Thaddeus, P.: 1971, Astrophys. J. 168, L67.

Lynds, B. T.: 1962, Astrophys. J. Suppl. 7, 1.

Palmer, P., Zuckerman, B., Buhl, D., and Snyder, L. E.: 1969, Astrophys. J. 156, L147. 